\title{
HYDROLYTIC CLEAVAGE OF THE PYRIMIDINE RING IN 2-ARYL-[1,2,4]TRIAZOLE[1,5-c]QUINAZOLINES: PHYSICO-CHEMICAL PROPERTIES AND THE HYPOGLYCEMIC ACTIVITY OF THE COMPOUNDS SYNTHESIZED
}

\author{
S.V.Kholodnyak, K.P.Schabelnyk, G.O.Zhernova, T.Yu.Sergeieva, V.V.Ivchuk, \\ O.Yu.Voskoboynik, S.I.Kovalenko, S.D.Trzhetsinskii, S.I.Okovytyy, S.V.Shishkina \\ Zaporizhzhia State Medical University \\ Dnepropetrovsk National University \\ Kryvyi Rih National University \\ SSI "Institute for Single Crystals", National Academy of Sciences of Ukraine
}

Key words: 2-aryl-[1,2,4]triazolo[1,5-c]quinazolines; hydrolytic cleavage; hypoglycemic activity

It has been shown that 2-aryl-[1,2,4]triazolo[1,5-c]-quinazolines under the action of nucleophilic agents (hydrazine hydrate, sodium hydroxide, sodium methoxide, hydrochloric acid) undergo hydrolytic cleavage followed by formation of [2-(3-aryl-1H-1,2,4-triazol-5-yl)-phenyl]amines. The rational synthetic protocols for the compounds mentioned above, namely heating in the hydrochloric acid solution at $90-95^{\circ} \mathrm{C}$ for $60 \mathrm{~min}$, have been proposed. It has been found that substituents in position 2 of the triazoloquinazoline moiety do not significantly affect duration of the reaction and the yields of products. Purity and the structure of the compounds synthesized have been proven by the corresponding physico-chemical methods, namely: elemental analysis, LC-MS, ${ }^{1} \mathrm{H},{ }^{13} \mathrm{C} N M R$-spectrometry and $X$-ray structural study. The azole-azole prototropic tautomery has been substantiated using physicochemical analytical methods. According to the data obtained in gas or DMSO medium compounds 2 exist as tautomer $A$ or $C$, while in the crystal lattice the anilines mentioned exist as A-form. It has been determined that 2-(3-aryl-1H-1,2,4-triazol-5-yl)phenyl]amines $(2.1,2.8,2.14)$ in the dose of $10 \mathrm{mg} / \mathrm{kg}$ are as good as reference-drugs Metformin (in the doses of 50 and $200 \mathrm{mg} / \mathrm{kg}$ ) and Gliclazide (in the dose of $50 \mathrm{mg} / \mathrm{kg}$ ) by their hypoglycemic activity when assessing specific pharmacological activities in oral glucose tolerance test (OGTT), rapid insulin and adrenaline test models.

Recent publication describes hydrolytic cleavage of azole and azine condensed cycles under the action of nucleophiles $[1,3,6,4,9,11,12,15,17,20,25,26,28$, 29]. It is known that kinetics of the reactions mentioned depends on basic properties of nucleophilic reagents. Strong nucleophiles (hydroxides and alkoxides of alkaline metals, hydrazine hydrate) easily react with the given substrates in the water or alcohol-water medium for $1 \mathrm{~h}$ and form products with high yields $[4,15,20,25,26$, $28,29]$. Moreover, water may play the role of a nucleophile in hydrolytic cleavage reactions. In this case the reaction proceeds for $1 \mathrm{~h}$ and needs acidic catalysis $[1,6]$. It has been noted that cleavage of the pyridine cycle of 2 -aryl-[1,2,4]triazolo[1,5-c]quinazoline is insufficiently known in spite of potentially bioactive products of this transformation. In addition, products of the reactions mentioned may take a worth place in the process of development of approaches for forming new heterocyclic systems and drug discovery [2, 8, 13, 18, 19, 23, 24].

Moreover, we found reports where the hypoglycemic activity of derivatives of 1,2,4-triazole were described. Thus, A.K.Mohammed Iqbal and co-authors discussed in their research the hypoglycemic and hypolipidemic action of novel compounds, which molecules contained the thiazolidone fragment attached to the triazole cycle via phenoxyethenthiol "linker" groups [14]. 4-Methyl-3(R-phenyl-,1-methyl-1H-indol-4(5)-yl)-5-(R-phenyl-)$4 H-1,2,4$-triazoles as selective inhibitors of $11 \beta$-hydroxysteroid dehydrogenase 1 were described as prospective glucose-lowering agents by Susan D. Aster and co-authors [7], and "ELI LILLY and COMPANY" applied for patent series of 2- $\mathrm{R}_{1}-4-\mathrm{R}_{2}-5$-alkaryl-(alkheteryl-, aryl-(heteryl-) oxy-, aryl-(heteryl-)thio-, aryl-(heteryl-)amino-)-2,4-dihydro-3H-1,2,4-triazol-3-ones(thiones) with high affinity to nuclear hormonal receptors. They may be used for treating diabetes, cardiovascular disorders, obesity, Xsyndrome and gastrointestinal disorders [21, 22], etc.

Thus, the aims of the present research were to study the peculiarities of the given type of the reaction, elucidation of the effect of $o-, m-, p$-substituted aryl moiety in position 2 of $[1,2,4]$ triazolo[ $[1,5-c]$ quinazolines on the process of the pyrimidine cycle hydrolytic cleavage, as well as the hypoglycemic action of the compounds synthesized.

\section{Experimental Part}

\section{Chemistry}

1.1. General method. Melting points were determined in open capillary tubes and were uncorrected. The ele- 
mental analyses $(\mathrm{C}, \mathrm{H}, \mathrm{N}, \mathrm{S})$ were performed using an ELEMENTAR vario EL Cube analyzer (USA). Analyses were indicated by the symbols of the elements or functions within $\pm 0.3 \%$ of the theoretical values. ${ }^{1} \mathrm{H}$ NMR spectra $(400 \mathrm{MHz})$ and ${ }^{13} \mathrm{C}$ NMR spectra $(100 \mathrm{MHz})$ : were recorded on a Varian-Mercury 400 (Varian Inc., Palo Alto, CA, USA) spectrometer with TMS as an internal standard in DMSO- $\mathrm{d}_{6}$ solution. LC-MS were recorded using the chromatography/mass spectrometric system consisting of high performance liquid chromatographer "Agilent 1100 Series" (Agilent, Palo Alto, CA, USA) equipped with a diode-matrix and a mass-selective detector "Agilent LC/MSD SL" (atmospheric pressure chemical ionization - APCI). Electron impact mass spectra (EI-MS) were recorded on a Varian $1200 \mathrm{~L}$ instrument at $70 \mathrm{eV}$ (Varian, USA).

Substances 1.1-1.16 were synthesized according to the procedures reported [16]. Other starting materials and solvents were obtained from commercially available sources and used without additional purification.

1.2. The general procedure for the synthesis of [2-(3aryl-1H-1,2,4-triazole-5-yl)penyllamines (2.1-2.17). Add 1-2 $\mathrm{ml}$ of concentrated hydrochloric acid to $10 \mathrm{mmol}$ of 2-aryl-[1,2,4]triazole[1,5-c]quinazolines (1.1-1.17) in $10 \mathrm{ml}$ of the water-alcohol mixture (1:1). Reflux the mixture obtained for $60 \mathrm{~min}$. Then dilute the mixture with water and add 5\% solution of sodium acetate to form the solution with $\mathrm{pH}$ 5-6. Filter the precipitates of compounds 2.12.17 and dry. Crystallize the compounds from methanol.

[2-(3-Phenyl-1H-1,2,4-triazole-5-yl)phenyl]amine (2.1). EI-MC, $m / z\left(\mathrm{I}_{\text {rel }}, \%\right): 237(15.6), 236\left(\mathrm{M}^{+}\right.$. 100), 207(6.0), 119(9.7), 118(21.7), 105(7.0), 104(38.6), 103 (4.0), 91(17.4), 90(7.2), 78(9.0), 77(27.1),63(5.1), 51(9.1).

\{2-[3-(2-Bromophenyl)-1H-1,2,4-triazole-5-yl]phenyl \}amine (2.4). EI-MC, $m / z\left(\mathrm{I}_{\mathrm{rel}}, \%\right): 317(13.2), 316(97.7)$, 315(12.9), 314( $\left.\mathrm{M}^{+*} .100\right), 236(7.4), 198(8.3), 196(9.6)$, 133(7.2), 131(5.7), 129(8.8), 119(5.8), 118(14.1), 117(6.7), 106(5.5), 105(11.8), 104(92.1), 103(31.2), 102(17.4), 91(14.5), 90(21.8), 89(14.0), 88(7.3), 85(5.4), 79(7.1), 78(22.5), 77(38.3), 76(13.1), 75(8.0), 65(9.2), 64(7.9), 63(11.6), 62(6.1), 52(7.0), 51(15.5).

\{2-[3-(2-Methoxyphenyl)-1H-1,2,4-triazole-5-yl]phenyl $\}$ amine (2.6). EI-MC, $m / z\left(\mathrm{I}_{\mathrm{rel}}, \%\right)$ : 267(16.9), 266( $\mathrm{M}^{+}$. 100), 265(8.2), 252(8.1), 248(6.3), 237(6.7), 236(9.3), 223(10.0) 146(9.3), 133(6.6), 132(5.5), $119(24.3), 118(35.9)$, 105(19.6), 104(48.6), 103(5.8), 102(5.1), 92(5.8), 91(19.1), 90(9.2), 85(9.1), 83(11.8), 79(7.4), 78(16.1), 77(38.8), 76(6.6), 65(8.6), 64(6.1), 63(6.4), 51(15.5).

\{2-[3-(4-Methylphenyl)- $1 H$-1,2,4-triazole-5-yl]phenyl amine (2.12). EI-MC, $m / z\left(\mathrm{I}_{\text {rel }}, \%\right): 251(16.3), 250\left(\mathrm{M}^{+}\right.$. 100), 221(7.8), 133(7.3), 132(18.8), 131(11.8), $119(1.8)$, 118(7.2), 105(9.9), 104(49.6), 91(20.3), 90(5.3), 78(12.4), 77(18.5), 65(7.5), 51(7.7); EI-MC. $m / z\left(\mathrm{I}_{\mathrm{rel}} . \%\right)=267(18.5)$, 266( $\left.\mathrm{M}^{+*} .100\right), 223(7.0), 148(10.5), 133(30.5), 119(5.6)$, 118(5.2), 105(17.9), 104(40.5), 91(8.1), 90(5.8), 78(12.6), 77(16.3), 76(5.7), 65(5.4), 63(5.1), 51(9.4).

\{2-[3-(4-Fluorophenyl)-1H-1,2,4-triazole-5-yl]phenyl $\}$ amine (2.13). EI-MC, $m / z\left(\mathrm{I}_{\text {rel }}, \%\right)=255(15,0), 254\left(\mathrm{M}^{+*}\right.$, 100), 137(9,5), 136(19,6), 109(18,4), 105(8,0), 104(36,9), $95(10,1), 78(9,3), 77(13,2), 51(6,8)$.
\{2-[3-(4-Chlorophenyl)-1H-1,2,4-triazole-5-yl]phenyl \}amine (2.14). EI-MC, $m / z\left(\mathrm{I}_{\text {rel }}, \%\right): 272(37.9), 271(14.1)$, 270( $\left.\mathrm{M}^{+*} .100\right), 154(9.6), 153(9.3), 152(21.8), 129(4.4)$, 127(5.3), 125(15.7), 119(7.2), 118(8.3), 111(11.2), 105(12.6), 104(63.4), 103(8.6), 102(7.5), 91(10), 90(19.1), 89(7.6), 85(8.3), 83(6.3), 79(6.6), 78(17.1), 77(26.6), 76(8.3), 75(10.7), 71(5.5), 69(9.4), 65(6.2), 63(9.8), 57(8.1), 55(6.2), 52(6.2), 51(10.8).

(2-\{3-[4-(Trifluoromethyl)phenyl]-1H-1,2,4-triazole-5-yl]phenyl $\}$ amine (2.16). EI-MC, $m / z\left(\mathrm{I}_{\text {rel }}, \%\right)=$ 305(17.5), 304( $\left.\mathrm{M}^{+*} .100\right), 187(10.1), 186(10.7), 145(9.5)$, 119(8.3), 118(11.2), 105(9.0), 104(51.4), 78(13.4), 77(20.0), $65(5.2), 51(10.2)$

\{2-[3-(4-Methoxyphenyl)-1H-1,2,4-triazole-5-yl]phenyl $\}$ amine (2.17). EI-MC, $m / z$ ( $\left.\mathrm{I}_{\text {rel }}, \%\right)$ : 267(18.5), 266(M ${ }^{+*}$. 100), 223(7.0), 148(10.5), 133(30.5), 119(5.6), 118(5.2), 105(17.9), 104(40.5), 91(8.1), 90(5.8), 78(12.6), 77(16.3), 76(5.7), 65(5.4), 63(5.1), 51(9.4).

1.3. X-Ray diffraction study of 2.16. The colourless crystals of $2.16\left(\mathrm{C}_{15} \mathrm{H}_{11} \mathrm{~N}_{4} \mathrm{~F}_{3}\right)$ are orthorhombic. At $293 \mathrm{~K}$ $\mathrm{a}=7.745(2), \mathrm{b}=11.435(2), \mathrm{c}=30.528(7) \AA, \mathrm{V}=2704(1) \AA^{3}$, $\mathrm{Mr}=304.28, \mathrm{Z}=8$, space group Pbca, $\mathrm{d}_{\text {calc }}=1.495 \mathrm{~g} / \mathrm{cm}^{3}$, $\mu\left(\mathrm{MoK}_{\sigma}\right)=0.122 \mathrm{~mm}^{-1}, \mathrm{~F}(000)=1248$. Intensities of 16607 reflections ( 2380 independent, $\left.R_{\text {int }}=0.194\right)$ were measured on a "Xcalibur-3" diffractometer (graphite monochromated $\mathrm{MoK}_{\alpha}$ radiation, CCD detector, $\omega$-scaning, $2 \Theta \max =50^{\circ}$ ). The structure was solved by the direct method using SHELXTL package [27]. Positions of the hydrogen atoms were located from electron density difference maps and refined by the "riding" model with $\mathrm{U}_{\text {iso }}=1.2 \mathrm{U}_{\text {eq }}$ of the carrier atom. The hydrogen atoms of the amino and $\mathrm{NH}$ groups were refined in isotropic approximation. Full-matrix least-squares refinement against $\mathrm{F}^{2}$ in anisotropic approximation for non-hydrogen atoms using 2354 reflections was converged to $\mathrm{wR}_{2}=0.191$ $\left(\mathrm{R}_{1}=0.068\right.$ for 927 reflections with $\left.\mathrm{F}>4 \sigma(\mathrm{F}), \mathrm{S}=0.869\right)$. The final atomic coordinates and crystallographic data for molecule 2.16 were deposited from the Cambridge Crystallographic Data Centre, 12 Union Road, CB2 1EZ, UK (fax: +44-1223-336033; e-mail: deposit@ccdc.cam.ac.uk). They are available on request quoting the deposition numbers CCDC 1029407.

\section{Pharmacology}

2.1. Hypoglycemia activity test. The study of the hypoglycemic action was conducted using 120 male Wistar white rats (with the weight of 260-280 g., aged 3.5 months) from nursery of PE "Biomodelservice" (Kyiv, Ukraine). Experiments on animals were done according to bioethic principles [10]. Selected after quarantine the animals were divided by random sampling in groups of 6 male rats on the assumption of the absence of the external signs of diseases and homogeneity by weight $( \pm 15 \%)$. Experimental animals were not fed within $12 \mathrm{~h}$ before introduction of the compounds studied. The weight of all animals was measured before the experiments. The compounds studied were injected intragastrically using atraumatic probe as the water solution or a finely dispersed suspension stabilized by Tween 80 in the dose of $10 \mathrm{mg} / \mathrm{kg}$. Intact and control groups received equivalent volumes of water by the same way. The hypoglycemic 


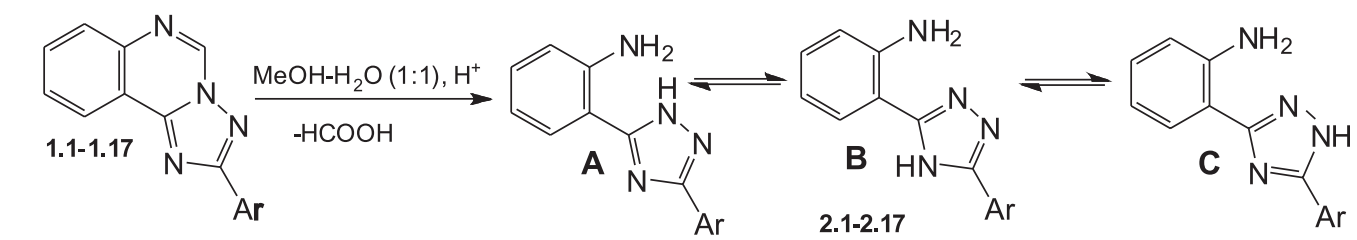

$\mathrm{Ar}=\mathrm{C}_{6} \mathrm{H}_{5}, 2-\mathrm{FC}_{6} \mathrm{H}_{4}, 2-\mathrm{ClC}_{6} \mathrm{H}_{4}, 2-\mathrm{BrC}_{6} \mathrm{H}_{4}, 2-\mathrm{CF}_{3} \mathrm{C}_{6} \mathrm{H}_{4}, 2-\mathrm{CH}_{3} \mathrm{OC}_{6} \mathrm{H}_{4}, 3-\mathrm{FC}_{6} \mathrm{H}_{4}, 3-\mathrm{ClC}_{6} \mathrm{H}_{4}$,

$3-\mathrm{BrC}_{6} \mathrm{H}_{4}, 3-\mathrm{CF}_{3} \mathrm{C}_{6} \mathrm{H}_{4}, 3-\mathrm{CH}_{3} \mathrm{OC}_{6} \mathrm{H}_{4}, 4-\mathrm{CH}_{3} \mathrm{C}_{6} \mathrm{H}_{4}, 4-\mathrm{FC}_{6} \mathrm{H}_{4}, 4-\mathrm{ClC}_{6} \mathrm{H}_{4}, 4-\mathrm{BrC}_{6} \mathrm{H}_{4}, 4-\mathrm{CF}_{3} \mathrm{C}_{6} \mathrm{H}_{4}, 4-\mathrm{CH}_{3} \mathrm{OC}_{6} \mathrm{H}_{4}$

Scheme. Hydrolytic cleavage of the pyrimidine ring in 2-aryl-[1,2,4]triazolo[1,5-c]-quinazolines and tautomeric transformation of [2-(3-aryl[1,2,4]triazol-5-yl)phenyl]amines.

action of the compounds synthesized was evaluated by changes in the glucose level before and after injection of the compounds studied. Measurements of the glucose level were carried out in 2, 4, 6 and $8 \mathrm{~h}$ after injection.

The primary insulin resistance was induced by a daily intramuscular injection of glucocorticoid, namely dexamethasone, in the dose of $0.125 \mathrm{mg} / \mathrm{kg}$ for 13 days $[5,30]$. The state of glucose homeostasis was evaluated by values of basal glycemia and carbohydrate tolerance determined by the oral test for glucose tolerance, rapid insulin test and adrenaline test $[5,30]$. Metformin in the doses of 50 and $200 \mathrm{mg} / \mathrm{kg}$ and Gliclazide in the dose of $50 \mathrm{mg} / \mathrm{kg}$ were used as reference drugs.

Statistical analysis was performed using a standard software complex, namely "Microsoft Office Excel 2003" and "STATISTICA ${ }^{\circledR}$ for Windows 6.0" (StatSoft Inc., № AXXR712D833214FAN5). For each estimated value in the arithmetic mean (M), and in the standard error of the mean $( \pm \mathrm{m})$ were determined. During verification of statistical hypotheses, in the null hypothesis was rejected if in the statistical criterion $\mathrm{p}<0.05$.

\section{Results and Discussion}

1. Chemistry. It was found that hydrolytic cleavage of 2-aryl-[1,2,4]triazolo[1,5-c]-quinazolines (1.1-1.17) occurred in the alcohol-water mixture medium in the presence of hydrochloric acid at $90-95^{\circ} \mathrm{C}$ for $60 \mathrm{~min}$ with the yields of $89.6-99.2 \%$ (Scheme). The synthetic protocols proposed are optimal for preparation of target compounds, while using hydrazine hydrate, sodium hydroxide and sodium methylate as reagents resulted not only in 2.1-2.17, but in products of greater decomposition of the initial molecule. Increase of the process time did not affect the yields of 2.1-2.17. As we considered, protonation of $\mathrm{N}_{6}$-atom, which caused increase of the positive charge at $\mathrm{C}_{5}$-atom, was significant for hydrolytic cleavage process. An adduct formed easily added the molecule of water (nucleophile), and it resulted in cleavage of $\mathrm{N}(4)-\mathrm{C}(5)$ bond, and followed by the nucleophilic attack of another molecule of water leading to elimination of formic acid and formation of 2.1-2.17. We also noted that in the conditions proposed there was no significant effect of the substituent's nature in position 2 of the triazoloquinazoline system on duration of the reaction and yields of final products.

Purity and the structure of the compounds synthesized were proven by the corresponding physico-chemical methods, namely: IR, ${ }^{1} \mathrm{H}$ and ${ }^{13} \mathrm{C}$ NMR, mass-spectrometry and X-ray structural analysis. In LC-MS spectra of 2.1-2.17 the high-intensive signals of quasi-molecular ions $[\mathrm{M}+1]$ and $[\mathrm{M}+2]$ corresponding to the calculated molecular weight were observed and definitely proved the fact of the pyrimidine cycle cleavage of the pyrimidine fragment in 1.1-1.17 (Tab. 1).

In ${ }^{1} \mathrm{H}$ NMR-spectra of compounds 2.1-2.17 the signals of aromatic protons of the aniline fragment with the proper chemical shift and multiplicity were observed, namely: doublet $\mathrm{H}^{3}$ at 8.11-7.78 ppm, triplet $\mathrm{H}^{4}-6.96-$ $6.51 \mathrm{ppm}$, triplet $\mathrm{H}^{5}$ - 7.57-7.08 ppm, doublet $\mathrm{H}^{6}$ - 7.16$6.74 \mathrm{ppm}$ (Table 2). Chemical shifts of the aryl fragment in position 3 depended on the position and the nature of substituents and were registered at 8.41-7.02 ppm. Among the peculiarities of ${ }^{1} \mathrm{H}$ NMR-spectra for 2.1-2.17 the signals caused by the presence of $\mathrm{NH}_{2}$-group were observed. Thus, the signals mentioned were present as a broad singlet at 6.75-6.26 ppm (2.1-2.3, 2.6-2.9, 2.12, $2.14,2.16)$ or absent $(2.4,2.5,2.10,2.11,2.13,2.15$, 2.17 ) as the result of exchange processes caused by prototropic azol-azol tautomery in 2.1-2.17 (Scheme). The absence $(2.8,2.11,2.12,2.14,2.15,2.17)$, doubling (2.1) or broadening $(2.2-2.7,2.9,2.10,2.13,2.16)$ of signal caused by NH-proton of the triazole cycle may be also explained by the abovementioned phenomenon. Thus, [2-(3-R-[1,2,4]triazol-5-yl)phenyl]amines (2.1-2.17) in DMSO- $\mathrm{d}^{6}$ are subjected to tautomery and may exist as $2 \mathrm{H}(\mathrm{A}), 1 \mathrm{H}(\mathrm{C})$ and $4 \mathrm{H}$ (B) forms (Scheme).

For additional verification of the structure of the compounds synthesized, as well as for tautomeric processes ${ }^{13} \mathrm{C}$ NMR-spectra were studied (Table 3 ). Signal correlations were carried out using the DEPT-method. It was found that signals caused by $\mathrm{C}^{3}$ and $\mathrm{C}^{5}$ atoms of the triazole cycle in molecules 2.1-2.17 were observed as broad singlets at $162.81-158.10 \mathrm{ppm}$ and 158.86 $150.79 \mathrm{ppm}$. It proves tautomeric transitions in DMSO-d $\mathrm{d}^{6}$ solutions. Besides, the signal of the atom of the aniline fragment deshielded as a result of the electron effect of the amino group $\mathrm{C}^{1}$ proves hydrolytic cleavage of the pyrimidine cycle.

Mass-spectra (EI) of compounds (2.1, 2.4, 2.6, 2.12, $2.13,2.14,2.16,2.17)$ were characterized by high molecular ions $[\mathrm{M}]^{+*}$. It is important to note that a donor or acceptor affecting the aryl substituent in position 2 had a significant influence on the directions of fragmentation. Thus, for compounds containing a donor substituent $(2.1,2.6,2.12,2.17)$ two parallel directions of fragmentation were observed. The first one was caused by breaking $\mathrm{N}(2)-\mathrm{C}(3)$ and $\mathrm{C}(5)-\mathrm{N}(1)$ bonds followed 
The physico-chemical data of [2-(3-aryl-1H-1,2,4-triazol-5-yl)phenyl]amine (2.1-2.17)

\begin{tabular}{|c|c|c|c|c|c|c|c|}
\hline Compd. & $\mathrm{R}$ & M.p., ${ }^{\circ} \mathrm{C}$ & Yield, \% & $\begin{array}{c}\text { Calculated, } \\
\mathrm{N}(\%)\end{array}$ & $\begin{array}{c}\text { Molecular } \\
\text { formula }\end{array}$ & Found, N (\%) & LC-MS, $m / z$ \\
\hline 2.1 & $\mathrm{H}$ & $188-190$ & 99.2 & 23.71 & $\mathrm{C}_{14} \mathrm{H}_{12} \mathrm{~N}_{4}$ & 23.73 & $237[\mathrm{M}+1], 238[\mathrm{M}+2]$ \\
\hline 2.2 & $2-\mathrm{F}$ & 195-197 & 89.6 & 22.04 & $\mathrm{C}_{14} \mathrm{H}_{11} \mathrm{FN}_{4}$ & 22.07 & $255[\mathrm{M}+1], 256[\mathrm{M}+2]$ \\
\hline 2.3 & $2-\mathrm{Cl}$ & $156-158$ & 92.8 & 20.70 & $\mathrm{C}_{14} \mathrm{H}_{11} \mathrm{CIN}_{4}$ & 20.73 & $271[\mathrm{M}+1], 273[\mathrm{M}+3], 274[\mathrm{M}+4]$ \\
\hline 2.4 & $2-\mathrm{Br}$ & 143-145 & 92.9 & 17.78 & $\mathrm{C}_{14} \mathrm{H}_{11} \mathrm{BrN}_{4}$ & 17.75 & $316[\mathrm{M}+1], 317[\mathrm{M}+2]$ \\
\hline 2.5 & $2-\mathrm{CF}_{3}$ & $201-203$ & 90.7 & 18.41 & $\mathrm{C}_{15} \mathrm{H}_{11} \mathrm{~F}_{3} \mathrm{~N}_{4}$ & 18.38 & $305[\mathrm{M}+1], 306[\mathrm{M}+2]$ \\
\hline 2.6 & $2-\mathrm{OCH}_{3}$ & 135-136 & 90.6 & 21.04 & $\mathrm{C}_{15} \mathrm{H}_{14} \mathrm{~N}_{4} \mathrm{O}$ & 21.06 & $267[M+1], 268[M+2]$ \\
\hline 2.7 & $3-\mathrm{F}$ & $215-217$ & 93.8 & 22.04 & $\mathrm{C}_{14} \mathrm{H}_{11} \mathrm{FN}_{4}$ & 22.01 & $255[\mathrm{M}+1], 256[\mathrm{M}+2]$ \\
\hline 2.8 & $3-\mathrm{Cl}$ & 196-197 & 95.5 & 20.70 & $\mathrm{C}_{14} \mathrm{H}_{11} \mathrm{CIN}_{4}$ & 20.67 & $271[\mathrm{M}+1], 273[\mathrm{M}+3], 274[\mathrm{M}+4]$ \\
\hline 2.9 & $3-\mathrm{Br}$ & 196-197 & 96.6 & 17.78 & $\mathrm{C}_{14} \mathrm{H}_{11} \mathrm{BrN}_{4}$ & 17.81 & $316[\mathrm{M}+1], 317[\mathrm{M}+2]$ \\
\hline 2.10 & $3-\mathrm{CF}_{3}$ & 237-239 & 94.2 & 18.41 & $\mathrm{C}_{15} \mathrm{H}_{11} \mathrm{~F}_{3} \mathrm{~N}_{4}$ & 18.45 & $305[\mathrm{M}+1], 306[\mathrm{M}+2]$ \\
\hline 2.11 & $3-\mathrm{OCH}_{3}$ & 177-179 & 95.5 & 21.04 & $\mathrm{C}_{15} \mathrm{H}_{14} \mathrm{~N}_{4} \mathrm{O}$ & 21.09 & $267[M+1], 268[M+2]$ \\
\hline 2.12 & $4-\mathrm{CH}_{3}$ & $166-168$ & 97.6 & 22.38 & $\mathrm{C}_{15} \mathrm{H}_{14} \mathrm{~N}_{4}$ & 22.35 & $251[\mathrm{M}+1], 252[\mathrm{M}+2]$ \\
\hline 2.13 & $4-\mathrm{F}$ & $209-211$ & 93.4 & 22.04 & $\mathrm{C}_{14} \mathrm{H}_{11} \mathrm{FN}_{4}$ & 22.01 & $255[\mathrm{M}+1], 256[\mathrm{M}+2]$ \\
\hline 2.14 & $4-\mathrm{Cl}$ & 289-291 & 97.3 & 20.70 & $\mathrm{C}_{14} \mathrm{H}_{11} \mathrm{CIN}_{4}$ & 20.73 & $271[\mathrm{M}+1], 273[\mathrm{M}+3], 274[\mathrm{M}+4]$ \\
\hline 2.15 & $4-\mathrm{Br}$ & $216-218$ & 98.4 & 17.78 & $\mathrm{C}_{14} \mathrm{H}_{11} \mathrm{BrN}_{4}$ & 17.76 & $305[\mathrm{M}+1], 306[\mathrm{M}+2]$ \\
\hline 2.16 & $4-\mathrm{CF}_{3}$ & $252-253$ & 94.1 & 18.41 & $\mathrm{C}_{15} \mathrm{H}_{11} \mathrm{~F}_{3} \mathrm{~N}_{4}$ & 18.39 & $305[\mathrm{M}+1], 306[\mathrm{M}+2]$ \\
\hline 2.17 & $4-\mathrm{OCH}_{3}$ & $206-207$ & 91.3 & 21.04 & $\mathrm{C}_{15} \mathrm{H}_{14} \mathrm{~N}_{4} \mathrm{O}$ & 21.01 & $267[\mathrm{M}+1], 268[\mathrm{M}+2]$ \\
\hline
\end{tabular}

Table 2

${ }^{1} \mathrm{H}$ NMR-spectra of [2-(3-aryl-1H-1,2,4-triazole-5-yl)phenyl]amine (2.1-2.17)

\begin{tabular}{|c|c|c|c|c|c|c|c|}
\hline \multirow{2}{*}{ Compd. } & \multicolumn{7}{|c|}{${ }^{1} \mathrm{H} N M R, \delta(\mathrm{ppm}), J(\mathrm{~Hz})$} \\
\hline & $\mathrm{NH}(\mathrm{bs})$ & $\mathrm{H}-3,(\mathrm{~d})$ & $\mathrm{H}-5,(\mathrm{t})$ & $\mathrm{H}-6,(\mathrm{~d})$ & $\mathrm{NH}_{2}$ (bs) & $\mathrm{H}-4,(\mathrm{t})$ & 3-Ar \\
\hline 2.1 & $14.48 / 14.20$ & $7.78(7.7)$ & $7.14(7.5)$ & $6.83(7.7)$ & 6.72 & $6.63(7.4)$ & $8.09\left(\mathrm{~d}, 7.0,2 \mathrm{H}, \mathrm{H}-2^{\prime}, 6^{\prime}\right), 7.49\left(\mathrm{~m}, 3 \mathrm{H}, \mathrm{H}-3^{\prime} 4^{\prime} 5^{\prime}\right)$ \\
\hline 2.2 & 14.36 & 7.87 & $7.15(7.2)$ & $6.85(8.1)$ & 6.75 & 6.65 (7.3) & $\begin{array}{l}8.11\left(\mathrm{t}, J=7.3 \mathrm{~Hz}, 1 \mathrm{H}, \mathrm{H}-6^{\prime}\right), 7.50\left(\mathrm{bs}, 1 \mathrm{H}, \mathrm{H}-4^{\prime}\right), \\
7.35\left(\mathrm{~m}, 2 \mathrm{H}, \mathrm{H}-3^{\prime}, 5^{\prime}\right)\end{array}$ \\
\hline 2.3 & 13.91 & $7.80(7.2)$ & $\begin{array}{l}7.33-7.22(\mathrm{~m}, \\
\left.2 \mathrm{H}, \mathrm{H}-5, \mathrm{H}-4^{4}\right)\end{array}$ & $6.74(8.1)$ & 6.26 & $6.58(7.3)$ & $\begin{array}{l}7.92\left(\mathrm{~d}, J=6.7 \mathrm{~Hz}, 1 \mathrm{H}, \mathrm{H}-6^{\circ}\right), 7.43(\mathrm{~d}, J=7.4 \mathrm{~Hz}, \\
\left.1 \mathrm{H}, \mathrm{H}-3^{\circ}\right), 7.04\left(\mathrm{t}, J=7.3 \mathrm{~Hz}, 1 \mathrm{H}, \mathrm{H}-5^{\circ}\right)\end{array}$ \\
\hline 2.4 & 14.40 & $\begin{array}{r}8.07-7.69(\mathrm{~m} \\
\left.3 \mathrm{H}, \mathrm{H}-3, \mathrm{H}-3^{\prime}, 6^{\prime}\right)\end{array}$ & $7.14(6.6)$ & $6.82(8.0)$ & 6.76 & $6.63(7.3)$ & $7.60-7.27\left(\mathrm{~m}, 2 \mathrm{H}, \mathrm{H}-3^{\prime}, \mathrm{H}-4^{\prime}\right)$ \\
\hline 2.5 & 14.49 & \multicolumn{6}{|c|}{$7.84\left(\mathrm{~m}, 4 \mathrm{H}, \mathrm{H}-3, \mathrm{H}^{\prime} 3^{`} 5^{`} 6^{\prime}\right), 7.17\left(\mathrm{t}, 1 \mathrm{H}, \mathrm{H}-4^{\prime}\right), 6.74(\mathrm{~m}, 3 \mathrm{H}, \mathrm{H}-4,5,6)$} \\
\hline 2.6 & 13.54 & $8.05(7.5)$ & $7.37(7.7)$ & $6.75(8.1)$ & 6.29 & $6.59(7.4)$ & $\begin{array}{l}8.24\left(\mathrm{~d}, J=6.9 \mathrm{~Hz}, 1 \mathrm{H}, \mathrm{H}-6^{\prime}\right), 7.05\left(\mathrm{~m}, 3 \mathrm{H}, \mathrm{H}-3^{\prime},\right. \\
\left.4^{\prime}, 5^{\prime}\right), 3.98\left(\mathrm{~s}, 3 \mathrm{H}, \mathrm{OCH}_{3}\right)\end{array}$ \\
\hline 2.7 & 14.42 & 7.94 (7.5) & $7.14(7.2)$ & $6.84(8.1)$ & 6.71 & $6.62(7.2)$ & $\begin{array}{l}7.83\left(\mathrm{~m}, 2 \mathrm{H}, \mathrm{H}-2^{\prime}, 6^{\prime}\right), 7.54(\mathrm{dd}, J=13.8,7.2 \mathrm{~Hz}, \\
\left.1 \mathrm{H}, \mathrm{H}-5^{\prime}\right), 7.26\left(\mathrm{t}, J=8.0 \mathrm{~Hz}, 1 \mathrm{H}, \mathrm{H}^{\prime} 4^{\prime}\right)\end{array}$ \\
\hline 2.8 & - & $7.88(7.8)$ & $7.11(7.6)$ & $6.82(8.2)$ & 6.71 & $6.60(7.4)$ & $\begin{array}{l}8.10\left(\mathrm{~s}, 1 \mathrm{H}, \mathrm{H}-2^{\prime}\right), 8.06(\mathrm{~d}, J= \\
\left.7.5 \mathrm{~Hz}, 1 \mathrm{H}, \mathrm{H}-6^{\prime}\right), 7.57-7.43\left(\mathrm{~m}, 2 \mathrm{H}, \mathrm{H}-4^{\circ}, 5^{\prime}\right) \\
\end{array}$ \\
\hline 2.9 & 14.15 & $7.75(6.2)$ & $7.08(7.2)$ & $6.79(7.8)$ & 6.26 & $6.59(7.3)$ & $\begin{array}{l}8.24\left(\mathrm{~s}, 1 \mathrm{H}, \mathrm{H}-2^{\prime}\right), 8.08\left(\mathrm{~d}, J=7.7 \mathrm{~Hz}, 1 \mathrm{H}, \mathrm{H}-6^{\prime}\right), \\
7.49\left(\mathrm{~d}, J=6.8 \mathrm{~Hz}, 1 \mathrm{H}, \mathrm{H}^{\prime} 4^{\prime}\right), 7.35(\mathrm{t}, J=7.5 \mathrm{~Hz}, \\
\left.1 \mathrm{H}, \mathrm{H}^{\circ} 5^{\prime}\right)\end{array}$ \\
\hline 2.10 & $14.61 / 14.30$ & $\begin{array}{l}\text { Overlaps on } \\
\text { 3-Ar }\end{array}$ & $7.13(7.2)$ & $6.83(7.2)$ & - & $6.61(7.4)$ & 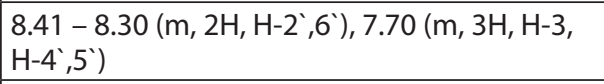 \\
\hline 2.11 & - & $7.83(5.8)$ & $7.08(7.5)$ & $6.94(8.0)$ & - & 6.59 (7.4) & $\begin{array}{l}7.64\left(\mathrm{~d}, J=7.6 \mathrm{~Hz}, 1 \mathrm{H}, \mathrm{H}-6^{\prime}\right), 7.59\left(\mathrm{~s}, 1 \mathrm{H}, \mathrm{H}-2^{\prime}\right) \\
7.36\left(\mathrm{t}, J=7.9 \mathrm{~Hz}, 1 \mathrm{H}, \mathrm{H}-5^{\prime}\right), 6.79(\mathrm{~d}, J=8.1 \mathrm{~Hz}, \\
\left.1 \mathrm{H}, \mathrm{H}^{\prime} 4^{\prime}\right), 3.83\left(\mathrm{~s}, 3 \mathrm{H}, \mathrm{OCH}_{3}\right)\end{array}$ \\
\hline 2.12 & - & $\begin{array}{c}\text { Overlaps on } \\
3-A r\end{array}$ & $7.25(6.9)$ & $7.06(7.4)$ & 6.22 & $6.88(6.9)$ & $\begin{array}{l}8.03\left(\mathrm{~m}, 3 \mathrm{H}, \mathrm{H}-3, \mathrm{H}-2^{\prime}, 6^{\prime}\right), 7.33(\mathrm{~d}, J=7.2 \mathrm{~Hz}, \\
\left.2 \mathrm{H}, \mathrm{H}-3^{\prime}, 5^{\circ}\right), 2.37\left(\mathrm{~s}, 3 \mathrm{H}^{\prime} \mathrm{CH}_{3}\right)\end{array}$ \\
\hline 2.13 & 14.36 & $7.83(7.3)$ & $7.15(7.3)$ & $6.85(7.8)$ & - & $6.64(7.0)$ & $\begin{array}{l}8.13\left(\mathrm{t}, J=6.7 \mathrm{~Hz}, 2 \mathrm{H},{\mathrm{H}-2^{\prime}}^{\prime}, 6^{\prime}\right), 7.34(\mathrm{t}, J= \\
\left.7.7 \mathrm{~Hz}, 2 \mathrm{H}, \mathrm{H}-3^{\prime}, 5^{\circ}\right) \\
\end{array}$ \\
\hline 2.14 & - & $8.11(7.0)$ & $7.57(7.0)$ & $7.16(7.0)$ & 6.84 & $4-6.63$ & $\begin{array}{l}8.30\left(\mathrm{~d}, J=7.1 \mathrm{~Hz}, 2 \mathrm{H}, \mathrm{H}-2^{\prime} 6^{\prime}\right), 7.85(\mathrm{~d}, 2 \mathrm{H}, J= \\
\left.7.1 \mathrm{~Hz}, 2 \mathrm{H}, \mathrm{H}-3^{`} 5^{\prime}\right)\end{array}$ \\
\hline 2.15 & - & $7.62(8.0)$ & $7.15(7.3)$ & $6.85(8.0)$ & - & $6.63(7.3)$ & $\begin{array}{l}7.85\left(\mathrm{~d}, J=8.0 \mathrm{~Hz}, 2 \mathrm{H},{\mathrm{H}-2^{\prime}}^{\prime}, 6^{\prime}\right), 7.66(\mathrm{~d}, J= \\
\left.7.9 \mathrm{~Hz}, 2 \mathrm{H}, \mathrm{H}-3^{\prime}, 5^{\prime}\right)\end{array}$ \\
\hline 2.16 & $14.60 / 14.23$ & $\begin{array}{l}\text { Overlaps on } \\
\text { 3-Ar }\end{array}$ & $7.11(7.6)$ & $6.82(7.5)$ & 6.73 & $6.60(7.4)$ & $\begin{array}{l}8.29\left(\mathrm{~d}, J=8.0 \mathrm{~Hz}, 2 \mathrm{H}, \mathrm{H}^{\prime} 2^{\prime} 6^{\prime}\right), 7.74(\mathrm{~m}, 3 \mathrm{H}, \mathrm{H}-3, \\
\left.\mathrm{H}^{\prime} 3^{\prime}, 5^{\prime}\right)\end{array}$ \\
\hline 2.17 & - & $8.04(7.6)$ & $7.29(7.5)$ & $7.13(7.9)$ & - & $6.96(7.0)$ & $\begin{array}{l}8.10\left(\mathrm{~d}, J=8.4 \mathrm{~Hz}, 2 \mathrm{H}, \mathrm{H}-2^{\prime}, 6^{\prime}\right), 7.09(\mathrm{~d}, J= \\
\left.8.5 \mathrm{~Hz}, 2 \mathrm{H}, \mathrm{H}-3^{\prime}, 5^{\prime}\right), 3.83\left(\mathrm{~s}, 3 \mathrm{H}, \mathrm{OCH}_{3}\right)\end{array}$ \\
\hline
\end{tabular}


${ }^{13} \mathrm{C}$ NMR-spectra of [2-(3-aryl-1H-1,2,4-triazole-5-yl)phenyl]amine (2.1-2.17)

\begin{tabular}{|c|c|c|c|c|c|c|c|c|c|}
\hline \multirow{2}{*}{ 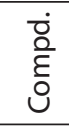 } & \multicolumn{9}{|c|}{${ }^{13} \mathrm{C}$ NMR, $\delta(\mathrm{ppm}), J(\mathrm{~Hz})$} \\
\hline & $\begin{array}{c}\text { triaz. } \\
\text { C-3 }\end{array}$ & $\begin{array}{c}\text { triaz. } \\
\text { C-5 }\end{array}$ & C-1 & C-5 & $\mathrm{C}-3$ & C-4 & C-6 & $\mathrm{C}-2$ & 3-Ar \\
\hline 2.1 & 160.73 & 154.13 & 147.44 & 129.20 & 126.44 & 116.50 & 115.28 & 108.96 & $131.40(\mathrm{C}-1,3,4,5), 127.47(\mathrm{C}-2,6)$ \\
\hline 2.2 & 162.81 & 154.66 & 146.83 & 129.72 & 124.59 & 116.02 & 115.16 & 109.26 & $\begin{array}{l}159.30 \text { (C-2), } 131.12 \text { (C-5), } 130.32 \text { (C-4), } 127.18 \text { (C-6), } \\
116.36 \text { (C3) }\end{array}$ \\
\hline 2.3 & 161.74 & 156.78 & 147.02 & 131.47 & 126.83 & 116.55 & 115.81 & 109.90 & $\begin{array}{l}132.48 \text { (C-2), } 130.64 \text { (C-1, 3), } 130.44 \text { (C-6), } 130.00 \text { (C-4), } \\
127.57 \text { (C-5) }\end{array}$ \\
\hline 2.4 & 159.40 & 154.54 & 146.80 & 130.33 & 126.99 & 116.01 & 115.11 & 107.95 & $\begin{array}{l}\text { 133.54 (C-3), } 131.54 \text { (C-1), } 131.27 \text { (C-5), } 131.01 \text { (C-6), } \\
127.47(\mathrm{C}-4), 121.00(\mathrm{C}-2)\end{array}$ \\
\hline 2.5 & 158.89 & 154.83 & 147.10 & 131.44 & 126.54 & 116.14 & 115.07 & 107.37 & $\begin{array}{l}132.27(\mathrm{C}-5), 132.06(\mathrm{C}-2), 130.81(\mathrm{C}-4), 130.30(\mathrm{C}-1) \\
129.26(\mathrm{C}-6), 126.67(\mathrm{C}-3), 123.87\left(\mathrm{q}, J=276.9 \mathrm{~Hz}_{1} \mathrm{CF}_{3}\right)\end{array}$ \\
\hline 2.6 & 160.58 & 150.79 & 146.33 & 130.71 & 120.30 & 115.53 & 115.03 & 112.80 & $\begin{array}{l}1(\mathrm{C}-4), 127.93(\mathrm{C}-5), \\
\left(\mathrm{OCH}_{3}\right)\end{array}$ \\
\hline 2.7 & 158.10 & 154.18 & 147.50 & 130.97 & 127.65 & 116.60 & 115.60 & \begin{tabular}{|l|}
109.28 \\
\end{tabular} & $\begin{array}{l}162.88 \text { (C-3), } 133.00 \text { (C-5), } 131.34 \text { (C-1), } 122.43 \text { (C-6), } \\
116.44 \text { (C-4), } 112.96(C-2)\end{array}$ \\
\hline 2.8 & 158.15 & 157.65 & 147.40 & 131.12 & 125.86 & 116.46 & 115.56 & 110.02 & $\begin{array}{l}134.01 \text { (C-3), } 132.99 \text { (C-1), } 130.61 \text { (C-5), } 129.17 \text { (C-2), } \\
127.77 \text { (C-4), } 124.88(C-6)\end{array}$ \\
\hline 2.9 & 159.32 & 155.84 & 147.28 & 131.58 & 127.33 & 116.53 & 115.56 & 108.54 & $\begin{array}{l}134.12(C-1), 130.68 \text { (C-5), } 130.46 \text { (C-2), } 129.04 \text { (C-4), } \\
125.12(C-6), 122.50(C-3)\end{array}$ \\
\hline 2.10 & 158.93 & 155.64 & 146.96 & 132.10 & 126.86 & 116.06 & 115.04 & 107.83 & $\begin{array}{l}130.27 \text { (C-1), } 129.79 \text { (C-5), } 129.33 \text { (C-6), } 124.99 \text { (C-4), } \\
123.78 \text { (C-3), } 122.06 \text { (C-2) }\end{array}$ \\
\hline 2.11 & 161.50 & 154.15 & 147.09 & 130.42 & 127.79 & 116.50 & 115.35 & 108.57 & \begin{tabular}{|l|}
$159.87(\mathrm{C}-3), 130.05(\mathrm{C}-1,5), 118.81(\mathrm{C}-6), 115.69(\mathrm{C}-4)$, \\
$111.58(\mathrm{C}-2), 55.43\left(\mathrm{OCH}_{3}\right)$
\end{tabular} \\
\hline 2.12 & 157.36 & 152.59 & 142.28 & 130.12 & 127.57 & 118.37 & 113.39 & 110.64 & $\begin{array}{l}139.31(\mathrm{C}-4), 130.12(\mathrm{C}-1), 129.32(\mathrm{C}-3,5), 126.03(\mathrm{C}-2,6), \\
20.91\left(\mathrm{CH}_{3}\right)\end{array}$ \\
\hline 2.13 & 159.35 & 155.65 & 146.72 & 130.02 & 127.17 & 116.11 & 115.26 & 108.00 & $162.68(C-4), 130.45(C-1), 128.09$ (C-2,6), $115.70(C-3,5)$ \\
\hline 2.14 & 161.94 & 157.48 & 146.98 & 130.48 & 125.65 & 116.05 & 115.09 & 108.76 & $134.10(C-1,4), 128.81(C-3,5), 126.41(C-2,6)$ \\
\hline 2.15 & 162.65 & 158.86 & 146.74 & 130.87 & 124.77 & 116.14 & 115.23 & 110.90 & $131.49(\mathrm{C}-3,5), 131.14(\mathrm{C}-2,6), 129.88(\mathrm{C}-1), 122.00$ (C-4) \\
\hline 2.16 & 160.38 & 155.44 & 146.81 & 130.33 & 126.74 & 116.08 & 114.96 & 107.78 & $\begin{array}{l}137.89(\mathrm{C}-1), 128.52(\mathrm{C}-4), 127.62(\mathrm{C}-3,5), 125.29(\mathrm{Ph} \mathrm{C}-2,6) \\
124.25\left(\mathrm{CF}_{3}\right)\end{array}$ \\
\hline 2.17 & 158.56 & 157.08 & 141.31 & 130.60 & 119.67 & 114.86 & 114.73 & 110.02 & $161.05(\mathrm{C}-4), 128.26(\mathrm{C}-2,6), 121.24 / 120.53(\mathrm{C}-1), 55.74\left(\mathrm{OCH}_{3}\right)$ \\
\hline
\end{tabular}

by formation of $\left[\mathrm{M}-\mathrm{N}_{2}\right]^{+\cdot}$ fragmental ion; the second one by breaking $\mathrm{N}(1)-\mathrm{N}(2)$ and $\mathrm{C}(3)-\mathrm{N}(4)$ followed by formation of $\left[\mathrm{M}-\mathrm{O}-\mathrm{NH}_{2} \mathrm{C}_{6} \mathrm{H}_{4} \mathrm{CN}\right]^{+\cdot}$ ion $(\mathrm{m} / \mathrm{z}$ 118). At the same time for compounds with an electron acceptor substituent $(2.4,2.13,2.14,2.16)$ only fragmentation by $\mathrm{N}(1)-\mathrm{N}(2)$ and $\mathrm{C}(3)-\mathrm{N}(4)$ bonds followed by formation of $\left[\mathrm{M}-\mathrm{O}-\mathrm{NH}_{2} \mathrm{C}_{6} \mathrm{H}_{4} \mathrm{CN}\right]^{+\cdot}$ ion $(\mathrm{m} / \mathrm{z} 118)$ was typical. Moreover, all compounds studied formed [M-o- $\left.\mathrm{NH}_{2} \mathrm{C}_{6} \mathrm{H}_{4}-\mathrm{CHN}\right]^{+*}$ ion ( $\mathrm{m} / \mathrm{z} 119)$. As we consider, formation of alternative ions with $\mathrm{m} / \mathrm{z} 119$ and 118 may be explained by existence of 1,2,4-triazol in the gas phase as two tautomer forms (A and C).

The spectral data of 10.1-10.6 presented allowed to prove the structure of the given compounds, but for undeniable elucidation of the nature of the molecule formed, as well as for clarification of a tautomeric form in the crystal state the X-ray structural study for compound 2.15 was used.

All non-hydrogen atoms of molecule 2.15 with the exception of fluorine atoms lie in the plane within $0.02 \AA$ (Fig. 1). Planar conformation is stabilized by the N4$\mathrm{H} 4 \mathrm{Nb} \ldots \mathrm{N} 3$ intramolecular hydrogen bond $(\mathrm{H}$... N 2.05 $\AA \mathrm{N}-\mathrm{H} \ldots \mathrm{N} 126^{\circ}$ ) and the $\mathrm{H} 14 \ldots \mathrm{N} 1$ and $\mathrm{H} 10 \ldots \mathrm{N} 3$ attractive interactions (H...N are $2.59 \AA$ and $2.60 \AA$, respectively, compared to the van der Waals radii sum [31]
$2.67 \AA$ ); it may not be considered as hydrogen bonds due to too small bond angles $\left(99^{\circ}\right.$ and $101^{\circ}$, respectively, Fig. 1). In the crystal phase molecules 2.15 form corrugated chains along the $\left[\begin{array}{lll}0 & 1 & 0\end{array}\right]$ crystallographic direction due to formation of $\mathrm{N} 2-\mathrm{H} . . . \mathrm{N} 4{ }^{\prime}(0.5-\mathrm{x}, \mathrm{y}-0.5, \mathrm{z})$ $\mathrm{H}$...N $2.01 \AA \mathrm{N}-\mathrm{H} . . . \mathrm{N} 161^{\circ}$. The participation of the lone pair of the $\mathrm{N} 4$ atom in this hydrogen bond results in pyramidal configuration of the amino group (the sum of bond angles centered at the $\mathrm{N} 4$ atom is $335^{\circ}$ ). The neighbouring chains are bonded by the N4-H4Na...N1' $(-\mathrm{x}, 0.5+\mathrm{y}, 0.5-\mathrm{z})$ intermolecular hydrogen bond $(\mathrm{H} . . \mathrm{N}$ $2.43 \AA \mathrm{N}-\mathrm{H} . . . \mathrm{N} 135^{\circ}$ ).

Thus, it was shown that 2 -aryl-[1,2,4]triazolo[ $[1,5-$ c]quinazolines under the action of nucleophilic agents

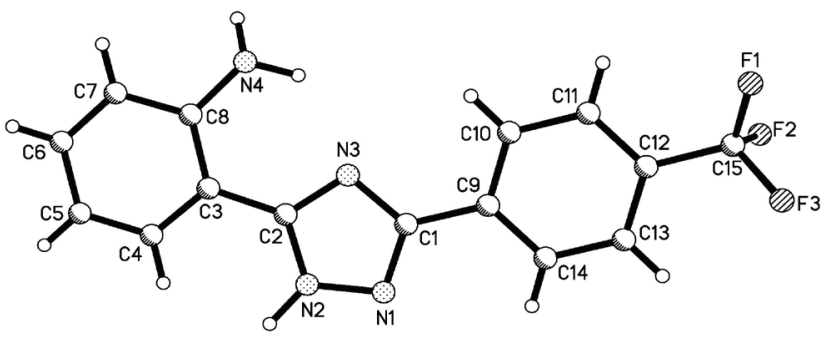

Fig. 1. The molecular structure of compound 2.15 according to X-ray diffraction data. 


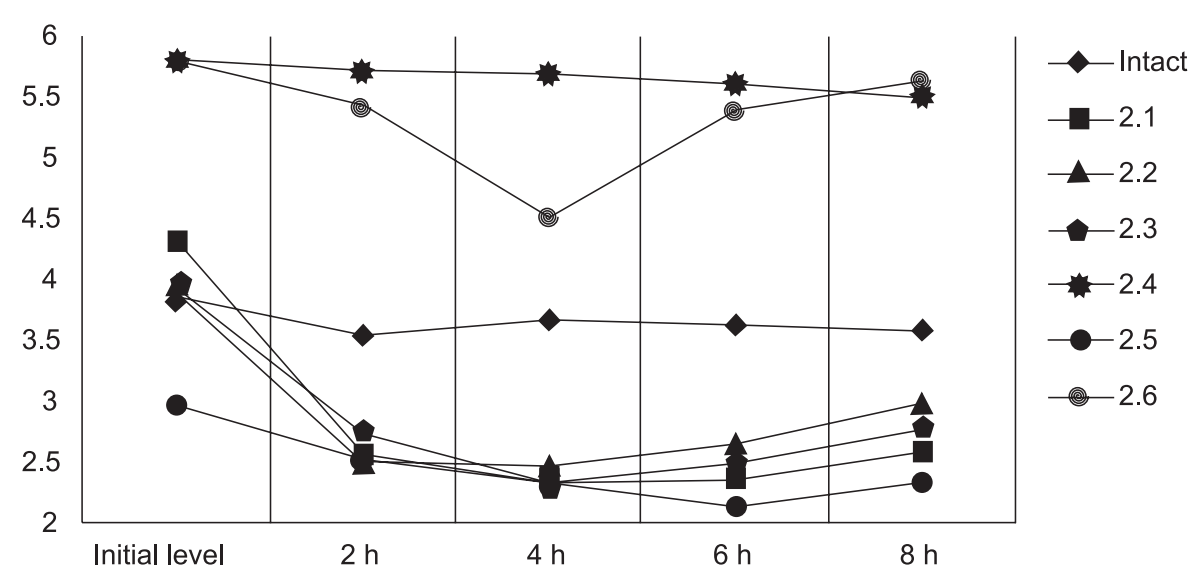

Fig. 2. The results of studying the hypoglycemic activity of [2-(3-phenyl-(2.1) 3-(2-R-phenyl)-(2.2-2.6)-1H-[1,2,4]triazolo-5-yl)phenyl]amines.

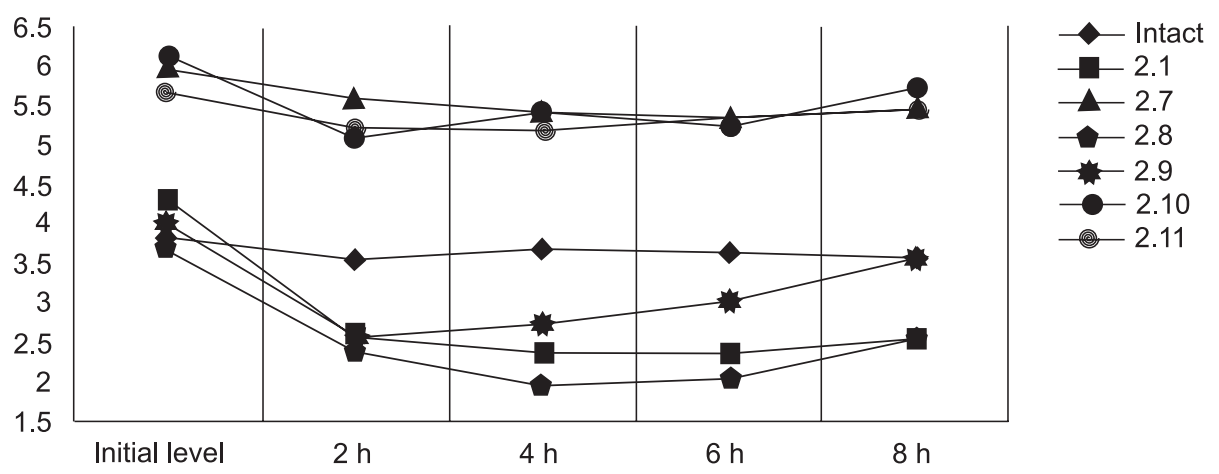

Fig. 3. The results of studying the hypoglycemic activity of [2-(3-phenyl- (2.1) 3-(3-R-phenyl)-(2.7-2.11)-1H-[1,2,4]triazolo-5-yl)phenyl]amines.

undergo hydrolytic cleavage followed by formation of [2-(3-aryl-1H-1,2,4-triazol-5-yl)phenyl]amines. The prototropic azole-azole tautomery has been also proven using physicochemical methods. We noted that the compounds synthesized exist as A-forms in crystals and as the equilibrium system of $\mathrm{A}$ and $\mathrm{C}$-forms in dimethylsulfoxide solutions and the gas phase.

\subsection{Hypoglycemic activity}

The results of the preliminary hypoglycemic activity assay are presented in Fig. 2-4. Thus, [2-(3-phenyl$1 H$-[1,2,4]triazolo-5-yl)phenyl]amine (2.1) decreases the blood glucose level of normoglycemic rats within the whole experiment. The glucose level was also lowered by injection of compounds $2.2,2.3$ and 2.5 containing fluorine, chlorine and trifluormethyl substituents in position 2 of the 3-phenyl fragment (Fig. 2). At the same time compounds containing 2-bromphenyl (2.4) and 2-methoxyphenyl (2.6) moieties in position 3 of the triazole cycle increased the glucose level within the whole experiment.

Translocation of substituents in the phenyl moiety from $o$ - (compounds 2.2-2.6) to $m$ - (2.7-2.11) position significantly changes the action of compounds (Fig. 3). Thus, most effective glucose lowering agents were compounds 2.8 and 2.9 containing chlorine and bromine. At the same time compounds with 3-fluoro- (2.7), 3-trifluoromethyl (2.10) and 3-methoxyphenyl (2.11) increased the blood glucose level.

Further modification of the compounds synthesized, namely translocation of substituents of the phenyl moiety from position $3(2.7-2.11)$ to position 4 (2.12-2.17), also led to ambiguous results. Experimental data have

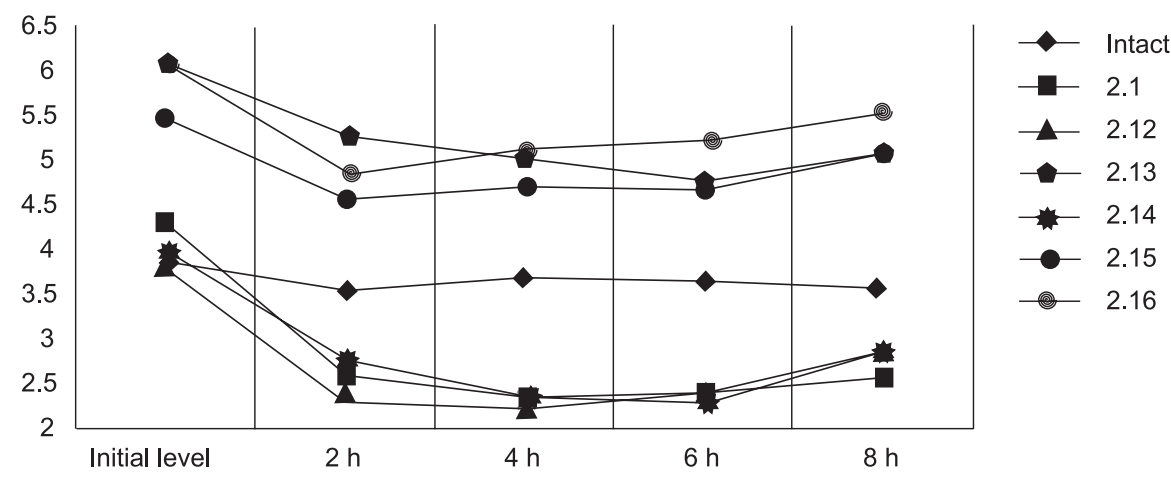

Fig. 4. The results of studying the hypoglycemic activity of [2-(3-phenyl-(2.1) 3-(4-R-phenyl)-(2.12-2.16)-1H-[1,2,4]triazolo-5-yl)phenyl]amines 
Results of the specific pharmacologic action evaluation of the compounds synthesized (oral test for glucose tolerance)

\begin{tabular}{|c|c|c|c|c|c|c|c|c|c|}
\hline Compd. & $\begin{array}{c}\text { Initial } \\
\text { glucose } \\
\text { level }\end{array}$ & $\begin{array}{l}\text { Glucose } \\
\text { level in } \\
15 \mathrm{~min}\end{array}$ & $\%$ increase & $\begin{array}{l}\text { Glucose } \\
\text { level in } \\
30 \text { min }\end{array}$ & $\%$ increase & $\begin{array}{l}\text { Glucose } \\
\text { level in } \\
60 \mathrm{~min}\end{array}$ & $\%$ increase & $\begin{array}{l}\text { Glucose } \\
\text { level in } \\
120 \text { min }\end{array}$ & $\%$ increase \\
\hline Control & $5.9 \pm 0.1$ & $7.7 \pm 0.3$ & $30.5 \pm 4.4$ & $8.7 \pm 0.2$ & $47.3 \pm 2$ & $9.4 \pm 0.1$ & $58.6 \pm 4.7$ & $6.8 \pm 0.2$ & $15.7 \pm 2.2$ \\
\hline Intact & $5.6 \pm 0.3$ & $7.5 \pm 0.3$ & $35.6 \pm 6.2$ & $7.8 \pm 0.4$ & $39.9 \pm 7.9$ & $6.8 \pm 0.3$ & $23.3 \pm 7.7^{\mathrm{a}}$ & $5.5 \pm 0.2$ & $-0.03 \pm 6.4^{a}$ \\
\hline Metformin 200 & $5.5 \pm 0.2$ & $6.8 \pm 0.1$ & $22.9 \pm 2.2$ & $7.5 \pm 0.1$ & $37.0 \pm 2.9^{\mathrm{a}}$ & $7.1 \pm 0.2$ & $29.6 \pm 1.7^{a}$ & $6.7 \pm 0.3$ & $22.2 \pm 1.8^{\mathrm{ab}}$ \\
\hline Metformin 50 & $5.5 \pm 0.3$ & $6.1 \pm 0.5$ & $9.3 \pm 2.7^{a}$ & $7.4 \pm 0.5$ & $33.0 \pm 1.5^{\mathrm{a}}$ & $7.6 \pm 0.6$ & $36.5 \pm 4.2^{\mathrm{a}}$ & $5.9 \pm 0.4$ & $5.9 \pm 3.0^{\mathrm{a}}$ \\
\hline Gliclazide 50 & $5.7 \pm 0.1$ & $7.7 \pm 0.1$ & $34.5 \pm 4.8$ & $7.9 \pm 0.1$ & $37.3 \pm 3.0^{\mathrm{a}}$ & $7.3 \pm 0.3$ & $27.8 \pm 3.02^{\mathrm{a}}$ & $6.2 \pm 0.1$ & $7.7 \pm 3.1$ \\
\hline 2.1 & $5.3 \pm 0.1$ & $7.1 \pm 0.1$ & $34.8 \pm 1.8$ & $8.1 \pm 0.1$ & $52.6 \pm 2.3$ & $7.1 \pm 0.2$ & $34.9 \pm 3.3^{\mathrm{a}}$ & $6.1 \pm 0.3$ & $15.1 \pm 4.5$ \\
\hline 2.8 & $6.1 \pm 0.1$ & $7.4 \pm 0.1$ & $20.8 \pm 0.9$ & $8.3 \pm 0.7$ & $35.7 \pm 8.8$ & $6.9 \pm 0.1$ & $13.7 \pm 0.8^{\mathrm{a}}$ & $6.7 \pm 0.2$ & $9.3 \pm 2.2$ \\
\hline 2.12 & $5.6 \pm 0.5$ & $8.1 \pm 0.4$ & $46.4 \pm 8.4$ & $8.7 \pm 0.6$ & $55.9 \pm 4.6$ & $9.3 \pm 0.7$ & $66.4 \pm 5.3$ & $7.7 \pm 0.5$ & $38.6 \pm 5.7^{a}$ \\
\hline 2.14 & $5.5 \pm 0.1$ & $7.0 \pm 0.3$ & $27.7 \pm 3.7$ & $7.5 \pm 0.5$ & $36.1 \pm 6.5$ & $7.7 \pm 0.3$ & $39.3 \pm 3.6^{a}$ & $5.8 \pm 0.2$ & $5.4 \pm 1^{\mathrm{a}}$ \\
\hline
\end{tabular}

a significant differences $(p \leq 0.05)$ compared to the control group of rats;

${ }^{b}$ significant differences $(p \leq 0.05)$ compared to the intact group of rats.

shown that some compounds $(2.12,2.14)$ significantly

Table 5 decrease the level of glucose, but other 2.13, 2.15 and 2.16 have quite opposite type of action (Fig. 4).

Thus, the preliminary screening conducted showed that among the compounds synthesized the most active were $2.1,2.8,2.12,2.14$. It was found that anilines 2.8 and 2.12 in the phenyl moiety contained a chlorine atom. At the next stage of our investigation the hypoglycemic action of compounds 2.1, 2.8, 2.12, 2.14 was studied using the glucocorticoid-induced insulin resistance model. Resistance to insulin was formed by the injection of dexamethasone.

Evaluation of the specific hypoglycemic action (oral test for glucose tolerance) in conditions of glucocorticoid-induced insulin resistance showed that compounds $2.1,2.8,2.14$ exhibited the hypoglycemic action, which was comparable to the activity of the reference-drugs "Metformin" (in the doses of 50 and $200 \mathrm{mg} / \mathrm{kg}$ ) and Gliclazide (in the dose of $50 \mathrm{mg} / \mathrm{kg}$, Tab. 4). At the same time compound 2.12 was inactive in the assay mentioned.

Further investigation of compounds 2.1, 2.8, 2.12, 2.14 in rats with glucocorticoid-induced insulin resistance using the rapid insulin test (Tab. 5) allowed to

Results of the specific pharmacologic action evaluation of the compounds synthesized (rapid insulin test)

\begin{tabular}{|c|c|c|c|}
\hline Compounds & $\begin{array}{c}\text { Initial glucose } \\
\text { level }\end{array}$ & $\begin{array}{c}\text { Glucose level } \\
\text { in } 30 \text { min }\end{array}$ & $\begin{array}{c}\text { \% decrease } \\
\text { of the glucose } \\
\text { level }\end{array}$ \\
\hline Control & $5.7 \pm 0.2$ & $4.3 \pm 0.1$ & $-24.6 \pm 1.1^{\mathrm{b}}$ \\
\hline Intact & $5.7 \pm 0.1$ & $2.3 \pm 0.1$ & $-60.4 \pm 0.9^{\mathrm{a}}$ \\
\hline Metformin 200 & $5.6 \pm 0.2$ & $3.6 \pm 0.1$ & $-34.6 \pm 3.7^{\mathrm{ab}}$ \\
\hline Metformin 50 & $5.2 \pm 0.2$ & $3.7 \pm 0.2$ & $-29.8 \pm 3.3^{\mathrm{b}}$ \\
\hline Gliclazide 50 & $5.4 \pm 0.2$ & $3.5 \pm 0.2$ & $-34.9 \pm 2.8^{\mathrm{ab}}$ \\
\hline 2.1 & $5.4 \pm 0.2$ & $4.0 \pm 0.1$ & $-25.7 \pm 3.6^{\mathrm{b}}$ \\
\hline 2.8 & $5.0 \pm 0.5$ & $3.6 \pm 0.8$ & $-45.4 \pm 1.3^{\mathrm{ab}}$ \\
\hline 2.12 & $5.0 \pm 0.3$ & $3.3 \pm 0.2$ & $-27.3 \pm 2.8^{\mathrm{b}}$ \\
\hline 2.14 & $5.4 \pm 0.4$ & $3.2 \pm 0.1$ & $-40.4 \pm 3.7^{\mathrm{ab}}$ \\
\hline
\end{tabular}

a - significant differences $(p \leq 0.05)$ compared to the control group of rats; ${ }^{b}$ - significant differences $(p \leq 0.05)$ compared to the intact group of rats.

obtain results, which were concordant with the data of the previous experiments and showed that compounds 2.8 and 2.14 exhibited the hypoglycemic action at the

Table 6

Results of the specific pharmacologic action evaluation of the compounds synthesized (adrenaline test)

\begin{tabular}{|c|c|c|c|c|c|}
\hline Compounds & Initial glucose level & $\begin{array}{c}\text { Glucose level } \\
\text { in } 30 \text { min }\end{array}$ & $\begin{array}{c}\text { \% increase of the } \\
\text { glucose level }\end{array}$ & $\begin{array}{c}\text { Glucose level } \\
\text { in 30 min }\end{array}$ & $\begin{array}{c}\text { \% increase of the } \\
\text { glucose level }\end{array}$ \\
\hline Control & $5.1 \pm 0.1$ & $12.4 \pm 0.5$ & $144.2 \pm 7.9^{\mathrm{b}}$ & $16.7 \pm 0.2$ & $228.9 \pm 6.1^{\mathrm{b}}$ \\
\hline Intact & $5.6 \pm 0.2$ & $11.5 \pm 0.4$ & $104.8 \pm 2.7^{\mathrm{a}}$ & $13.7 \pm 0.6$ & $144.3 \pm 5.7^{\mathrm{b}}$ \\
\hline Metformin 200 & $6.7 \pm 0.2$ & $9.6 \pm 0.2$ & $42.2 \pm 2.8^{\mathrm{ab}}$ & $10.9 \pm 0.1$ & $63.3 \pm 4.9^{\mathrm{ab}}$ \\
\hline Metformin 50 & $5.5 \pm 0.1$ & $6.7 \pm 0.2$ & $21.2 \pm 3.1^{\mathrm{ab}}$ & $10.6 \pm 0.2$ & $92.2 \pm 4.9^{\mathrm{ab}}$ \\
\hline Gliclazide 50 & $6.0 \pm 0.1$ & $7.7 \pm 0.2$ & $28.4 \pm 5.9^{\mathrm{ab}}$ & $10.1 \pm 0.2$ & $66.4 \pm 4.4^{\mathrm{ab}}$ \\
\hline 2.1 & $5.1 \pm 0.1$ & $9.8 \pm 0.3$ & $93.4 \pm 1.8^{\mathrm{ab}}$ & $8.8 \pm 0.7$ & $73.5 \pm 10.3^{\mathrm{ab}}$ \\
\hline 2.8 & $6.4 \pm 0.1$ & $7.9 \pm 0.3$ & $23.5 \pm 2.9^{\mathrm{ab}}$ & $9.2 \pm 0.4$ & $43.8 \pm 2.8^{\mathrm{ab}}$ \\
\hline 2.12 & $7.1 \pm 0.4$ & $13.8 \pm 2.4$ & $94.5 \pm 24.4$ & $17.5 \pm 3.3$ & $147.3 \pm 33.8$ \\
\hline 2.14 & $6.3 \pm 0.3$ & $9.4 \pm 0.5$ & $50.5 \pm 12.9^{\mathrm{ab}}$ & $12.2 \pm 0.7$ & $93.9 \pm 5.2^{\mathrm{ab}}$ \\
\hline
\end{tabular}

a - significant differences $(p \leq 0.05)$ compared to the control group of rats; ${ }^{b}$ - significant differences $(p \leq 0.05)$ compared to the intact group of rats. 
level similar to the reference drugs. Thus, the hypoglycemic activity of [2-(3-chloro- $1 H$-[1,2,4]-triazole-5-yl) phenyl]aniline (2.8) exceeded the action of Gliclazide by $10.5-15.6 \%$.

We noted that [2-(3-chloro- $1 H$-[1,2,4]-triazole-5-yl) phenyl]amine (2.8) exhibited a high hypoglycemic activity in rats with glucocorticoid-induced insulin resistance using the adrenaline test. Thus, compound $2.8 \mathrm{ex}-$ ceeded the action of the reference drugs by $19.5-48.4 \%$ (Tab. 6).

\section{CONCLUSIONS}

According to the data of the specific pharmacologic action evaluation of the compounds synthesized (oral test for glucose tolerance, rapid insulin test and adrenaline test) the hypoglycemic action of 2-(3-aryl-H-1,2,4-triazol-5-yl)phenyl]amines $(2.1,2.14)$ in the dose of $10 \mathrm{mg} / \mathrm{kg}$ is not inferior to the action of the reference drugs Metformin (in the doses of 50 and $200 \mathrm{mg} / \mathrm{kg}$ ) and Gliclazide (in the dose of $50 \mathrm{mg} / \mathrm{kg}$ ), moreover compound $2.8 \mathrm{ex}$ ceeds the activity of the drugs mentioned above.

\section{REFERENCES}

1. Білий А.К., Коваленко С.І., Приходько О.Б. та ін. // Запорізький мед. журн. - 2013. - №2 (77). - С. 83-86.

2. Волошина В.О., Литвиненко М.О., Сапегин І.Д. та ін. // Мед. хім. - 2010. - Т. 12, №3 (44). - С. 98-107.

3. Уломский Е.Н., Воронин В.В., Русинов В.Л. и др. // Известия АН. Серия химическая. - 2001. - №4. - С. 655-661.

4. Швайка О.П., Артемов В.Н. // Успехи химии. - 1972. - Т. XLI (10). - С. 1788-1822.

5. Akinmokun A., Selby P.L., Ramaya K. et al. // Diab. Med. - 1992. - Vol. 9. - P. 432-437.

6. Antipenko L.N., Karpenko A.V., Kovalenko S.I. et al. // Chem. Pharm. Bull. - 2009. - Vol. 57 (6). - P. 580-585.

7. Aster S.D., Graham D.W., Kharbanda D. et al. // Bioorg. \& Med. Chem. Lett. - 2008. - Vol. 18. - P. 2799-2804.

8. Berest G.G., Voskoboynic A.Yu., Kovalenko S.I. et al. // ЖОФX. - 2010. - T. 8, вun. 3 (31). - C. 42-52.

9. Dalpiaz A., Bertolasi V., Ferretti V. et al. // J. Chem. Crystallogr. - 1997. - Vol. 27 (1). - P. 59-66.

10. European convention for the protection of vertebrate animals used for experimental and other scientific purpose: Council of Europe. - Strasbourg, 1986. - P. 52.

11. Francis J.E., Cash W.D., Barbaz B.S. et al. // J. Med. Chem. - 1991. - Vol. 34 (1). - P. 281-290. doi: 10.1021/ jm00105a044

12. Francis J.E., Cash W.D., Psychoyos S. et al. // J. Med. Chem. - 1988. - Vol. 31 (5). - P. 1014-1020.

13. Gerecke M., Kyburz E., Borer R. et al. // Heterocycles. - 1994. - Vol. 39 (2). - P. 693-721.

14. Iqbal M.A. K., Khan A.Y., Kalashetti M.B. et al. // Eur. J. of Med. Chem. - 2012. - Vol. 53. - P. 308-315.

15. Karpenko A.V., Kovalenko S.I., Shishkin O.V. // Tetrahedron. - 2009. - Vol. 65 (31). - P. 5964-5972.

16. Kovalenko S.I., Antypenko L.M., Bilyi A.K. et al. // Sci. Phar. - 2013. - Vol. 81 (2). - P. 359-391. doi:10.3797/ scipharm.1211-08

17. Maiboroda D., Babaev E. // J. Org. Chem. - 1997. - №62. - P. 7100-7105.

18. Moustafa H.M. // Phosphorus, Sulfur, and Silicon and the Related Elements. - 2000. - Vol. 164 (1). - P. 11-22.

19. Mustazza C., Borioni A., Sestili I. et al. // Chem. Pharm. Bull. -2006. - Vol. 54 (5). - P. 611-622.

20. Pat. USA 4020083. Filed: 08.07.1975, Posted: 27.04.1977; [електронний ресурс], режсим доступу: http:// www.freepatentsonline.com $/ 4020083 . \mathrm{html}$

21. Pat. USA 20090062358, Filed: 09.11.2001; Posted: 16.05.2002; [електронний ресурс], режим доступу: http://www. freepatentsonline.com/y2009/0062358.html.

22. Pat. WO 1996/13264 A1. Filed: 01.11.1994; Posted: 09.05.1996; [електронний ресурс], режим достуnу: http://www.freepatentsonline.com/WO1996013264A1.html.

23. Pat. WO/2006/063708, Filed: 05.12.2005; Posted: 22.06.2006; [електронний ресурс], режим доступу: http://www.freepatentsonline.com/WO2006063708A1.html.

24. Pat. WO/2007/042421, Filed: 02.10.2006; Posted: 19.04.2007; [електронний ресурс], режим доступу: http://www.freepatentsonline.com/WO2007042421A1.html.

25. Petric A., Tisler M., Stanovnik B. // Monatsh. Chem. - 1985. - Vol. 116. - P. 1309-1320.

26. Sergeieva T.Yu., Voskoboynik O.Yu., Okovytyy S.I. et al. // J. Phys. Chem. A. - 2014. - Vol. 118. - P. 1895-1905. doi.org/10.1021/jp4052616

27. Sheldrick G.M. // Acta Crystallogr., Sect. A 2008, A64, 112. doi:10.1107/S0108767307043930

28. Shishoo C.J., Devani M.B., Ullas G.V. et al. // J. Heterocyclic Chem. - 1987. - Vol. 24. - P. 1125-1131.

29. Voskoboynik A.Yu., Berest G.G., Skorina D.Yu. et al. // Chemistry \& Chemical Technol. - 2011. - Vol. 5 (2). - P. 129-132.

30. Weinstein S.P., Paquin T., Pritsker A. et al. // Diabetes. - 1995. - Vol. 44. - P. 441-445.

31. Zefirov Yu.V. // Kristallographiya (Russian). - 1997. - Vol. 42. - P. 936-958. 
ГІДРОЛІТИЧНЕ РОЗЩЕПЛЕННЯ ПІРИМІДИНОВОГО ЦИКЛУ

В 2-АРИЛ-[1,2,4]ТРИАЗОЛО[1,5-С]ХІНАЗОЛІНАХ: ФІЗИКО-ХІМІЧНІ ВЛАСТИВОСТІ ТА ГІПОГЛІКЕМІЧНА АКТИВНІСТЬ СИНТЕЗОВАНИХ СПОЛУК С.В.Холодняк, К.П.Шабельник, Г.О.Жернова, Т.Ю.Сергеєва, В.В.Івчук, О.Ю.Воскобойнік, С.І.Коваленко, С.Д.Тржецинский, С.І.Оковитий, С.В.Шишкіна

Ключові слова: 2-арил-[1,2,4]триазоло[1,5-с]хіназоліни; гідролітичне розщеплення;

гіпоглікемічна активність

Показано, що 2-арил-[1,2,4]триазоло[1,5-с]хіназоліни під дією нуклеофрільних реагентів (гідразин гідрат, гідроксид або мелилат натрію, кислота хлористоводнева) піддаються гідролітичному розщепленню з утворенням 2-(3-арил-1Н-1,2,4-триазол-5-іл)феніл]амінів. Запропоновані оптимальні умови синтезу: хлористоводнева кислота, спирто-водне середовище, температура $90-95^{\circ} \mathrm{C}$, тривалість 60 хвилин. Встановлено, що за запропонованих умов синтезу не спостерігається суттєвого впливу замісника положення 2 триазолохіназолінового циклу на тривалість реакції та виходи кінцевих продуктів. Індивідуальність синтезованих сполук підтверджена хроматомас-спектрометрією, будова - елементним аналізом, ${ }^{1} \mathrm{H},{ }^{13} \mathrm{C}$ ЯMP-, мас-спектрами та рентгеноструктурним аналізом. За допомогою фрізико-хімічних методів обгрунтована азол-азольна (прототропна) таутомерія синтезованих сполук. Показано, що в розчинах ДМСО та газовій фразі 2-(3-арил-1Н-1,2,4-триазол-5-іл)феніл]аміни переважно існують у вигляді A та C-фрорм, тоді як у кристалічній решітиі існують у вигляді А-срорми. Встановлено, що 2-(3-арил-1Н-1,2,4-триазол-5-іл)френіл]аміни (2.1, 2.8, 2.14) в дозі 10 ме/ка при визначенні специфрічної фрармакологічної активності, а саме оральному тесті толерантності до глюкози (ОТТГ)), короткому інсуліновому та адреналіновому тесті не поступаються за гіпоглікемічною активністю референс-препаратам «Метформіну» (доза 50 та 500 мг/кг) та «Гліклазиду» (доза 50 мг/ка).

\section{ГИДРОЛИТИЧЕСКОЕ РАСЩЕПЛЕНИЕ ПИРИМИДИНОВОГО ЦИКЛА}

\section{2-АРИЛ-[1,2,4]ТРИАЗОЛО[1,5-С]ХИНАЗОЛИНОВ: ФИЗИКО-ХИМИЧЕСКИЕ СВОЙСТВА И ГИПОГЛИКЕМИЧЕСКАЯ АКТИВНОСТЬ СИНТЕЗИРОВАННЫХ СОЕДИНЕНИЙ С.В.Холодняк, К.П.Шабельник, Г.А.Жернова, Т.Ю.Сергеева, В.В.Ивчук,} А.Ю.Воскобойник, С.И.Коваленко, С.Д.Тржецинский, С.И.Оковитый, С.В.Шишкина Ключевые слова: 2-арил-[1,2,4]триазоло[1,5-с]хиназолины; гидролитическое расщепление; гипогликемическая активность

В представленной работе показано, что 2-арил-[1,2,4]триазоло[1,5-с]хиназолины под действием нуклеофильных реагентов (гидразин гидрат, гидроксид натрия или метилат натрия, кислота хлористоводородная) подвергаются гидролитическому расщеплению с образованием 2-(3-арил-1Н-1,2,4-триазол-5-ил)фенил]аминов. Предложены оптимальные условия синтеза: хлористоводородная кислота, спирто-водная среда, температура 90-95 $\mathrm{C}$, продолжительность 60 минут. Установлено, что в предложенных условиях синтеза не наблюдается значительного влияния заместителя положения 2 триазинохиназолинового цикла на продолжительность реакции и выходы конечных продуктов. Индивидуальность синтезированных соединений подтверждена хроматомас-спетрометрией, строение - элементным анализом, ${ }^{1} \mathrm{H},{ }^{13} \mathrm{C}$ ЯMP-, масс-спектрами и рентееноструктурным анализом. С использованием фризико-химических методов обоснована азол-азольная (прототропная) таутомерия синтезированных соединений. Показано, что в растворах ДМСО и газовой фразе 2-(3-арил1Н-1,2,4-триазол-5-ил)феенил]амины главным образом существуют в виде A и C-фрорм, тогда как в кристаллической решетке в виде А-фрормы. Установлено, что 2-(3-арил-1Н-1,2,4триазол-5-ил)френил]амины $(2.1,2.8,2.14)$ в дозе 10 мг/ка при оценке гипогликемической активности в оральном тесте толерантности к глюкозе (ОТТГ), коротком инсулиновом и адреналиновом тесте не уступают по гипогликемической активности рефреренс-препаратам «Меторормину» (доза 50 и 500 мг/кг) и «Гликлазиду» (доза 50 мг/кг). 